\title{
Aplicação de PDCA no processo produtivo de dobradiças
}

Almir Pereira da Cruz Filho almirpereira402@gmail.com Centro Universitário ENIAC (ENIAC), Guarulhos, São Paulo, Brasil

Cristiano do Vale Noronha cristiano.v.noronhacummins@gmail.com Centro Universitário ENIAC (ENIAC) Guarulhos, São Paulo, Brasil

Renato de Brito Sanchez renatobritosanchez@gmail.com Centro Universitário ENIAC (ENIAC) (UMC) Mogi das Cruzes, São Paulo, Brasil

\author{
RESUMO
}

Devido à evolução nos sistemas de produção, ocasionado por conta dos avanços tecnológicos e melhoria nos sistemas de qualidade, é possível perceber que com o passar dos anos, muitas empresas investiram em maquinário de alta tecnologia. Porém às vezes, algumas destas empresas esquecem-se de utilizar uma ferramenta da qualidade para o acompanhamento dos seus processos produtivos, o que é de extrema importância para medir seu desempenho e identificar possíveis melhorias em seu processo. Assim sendo, foi escolhido como base para este estudo de caso a empresa que investiu em tecnologia de automação concentrada na montagem de dobradiças, que assim como outras indústrias moveleira também aderiram a automação na linha de produção. Visando o processo de montagem, foi identificado que mesmo com investimentos tecnológicos, é necessário a inserção de uma ferramenta de qualidade para o acompanhamento dos processos. Desta forma, através de demonstrativos de viabilidades em relação ao aumento da produção será exposto a eficiência de uma ferramenta da qualidade. Em um estudo geral, os objetivos a atingir com a automação e a monitoria do Controle de Qualidade (CQ) poderá enquadrar em dois grandes problemas, como refugos e retrabalhos.

PALAVRAS-CHAVE: PDCA. Produção de dobradiças. Check list PDCA. Processos PDCA. Folha de verificação PDCA. 


\section{INTRODUÇÃO}

A análise para este estudo de caso é baseada na aplicação de métodos de monitoramento aplicável nos processos de produção e montagem de dobradiças e tem como objetivo aplicar o ciclo PDCA na produção e montagem de dobradiças, verificar onde se encontra o gargalo que prejudica a montagem das peças. A identificação deste problema tem por objetivo inserir uma ferramenta da qualidade na produção de dobradiças, facilitando e eliminando problemas como refugos e retrabalho, assim fazendo com que a empresa consiga atingir sua meta de produção diária.

Os métodos que serão utilizados demonstrarão a capacidade dos processos em alcançar os resultados planejados. A melhoria abordada realça à organização, fazer a medição e monitoria das características do produto para verificar se os requisitos dele estão sendo atendidos. Isso deve ser realizado em fases de cada operação do processo de fabricação do produto, de acordo com as providências planejadas (CQ - Controle de Qualidade). "A fase mais importante no planejamento é identificar a problemática antes de inserir a melhoria e reduzir as não conformidades, para que não haja perca de tempo e nem dinheiro" (AGUIAR, 2002). As medições seriam realizadas em laboratórios e a partir dos resultados desenvolvidos gabaritos para que sejam feitas as comparações conforme os horários de inspeção estabelecidos pela qualidade e engenharia da empresa.

Identificado no processo de montagem de dobradiças da empresa, sendo processo manual, o qual gerava um acúmulo de pessoas, e um acabamento imperfeito acumulando assim peças não conformes e muitas vezes o desperdício de materiais em peças que não foram montadas corretamente. $\mathrm{Na}$ indústria moveleira, segundo Gorini (1998) "a competitividade depende não somente da eficiência dos processos produtivo, mas também da qualidade, do conforto, da facilidade de montagem e, sobretudo, dos modelos de móveis". Além disso, o processo produtivo se tornava lento, e não atendia totalmente a demanda da organização. Por exemplo, os montadores conseguiam liberar cerca de 7.000 mil peças/dia e a demanda da organização seria de que fossem liberadas cerca de 10.000 peças/dia. Sendo assim, a análise crítica foi baseada a partir de um aumento grande de refugos por não existir um acompanhamento adequado de uma ferramenta da qualidade na montagem e produção de dobradiças, a qual foi utilizado o método gerencial, e apresentado o PDCA como melhoria.

\section{MÉTODO}

Campos (2004) define o ciclo PDCA como, "O PDCA é um método de gerenciamento de processos ou de sistemas. É o caminho para se atingirem as metas atribuídas aos produtos dos sistemas empresariais." Analisando a definição de Campos, foi nomeado com o termo "método". A palavra "método" é originada pela união de dois termos gregos: meta + hodos, isto é, caminho para a meta. Sendo assim, segundo a própria definição da citação, o ciclo PDCA é "um caminho para se atingir uma meta" (CAMPOS, 2004). Conclui-se, portanto, que não é possível estabelecer um ciclo PDCA sem uma meta definida. O ciclo PDCA é definido como um checklist de quatro etapas. 
Figura 1 - O Ciclo PDCA

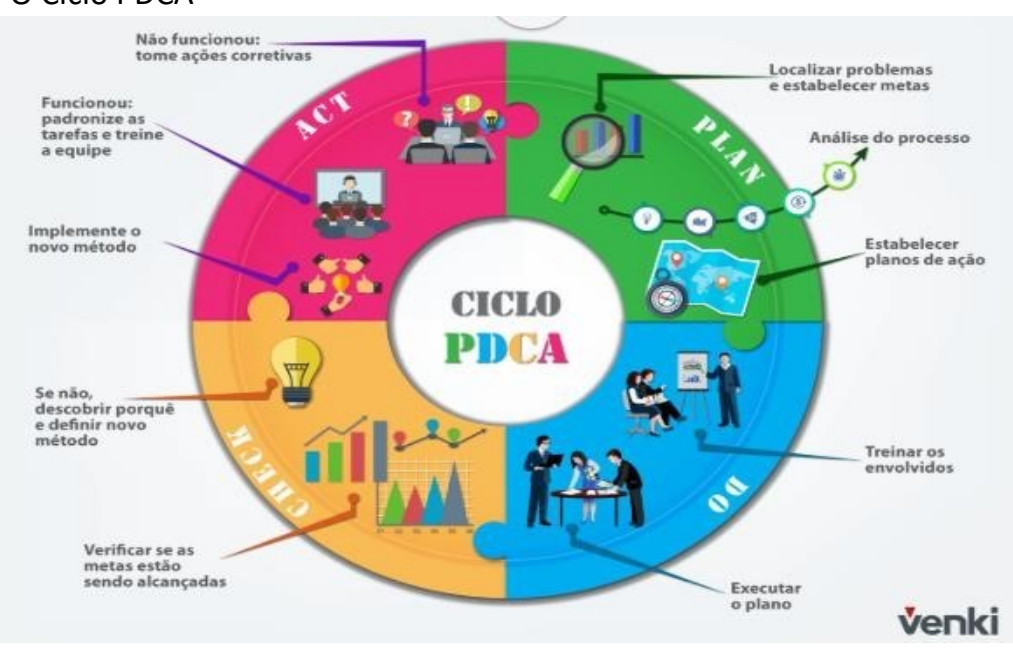

Fonte: Veyrat (2019)

"A natureza repetida e cíclica da melhoria contínua pode ser resumida no ciclo do PDCA, definido como uma sequência de atividades que são percorridas de maneira cíclica para melhoria das atividades" (SLACK, 2009). Isto permite que os processos sejam aproveitados de maneira efetiva, reduzindo o valor dos custos e aumentando a produtividade. Esse ciclo ininterrupto de mudança é representado na rampa de melhoria conforme mostrado na Figura 2. Usando o que foi aprendido em uma aplicação do ciclo PDCA, pode-se começar outro ciclo, em uma tentativa mais complexa, e assim sucessivamente. Sendo assim, o último ponto sobre o ciclo PDCA se torna o mais importante, onde o ciclo assumirá um novo começo (NASCIMENTO, 2011).

Figura 2 - Rampa de Melhoria do Ciclo PDCA

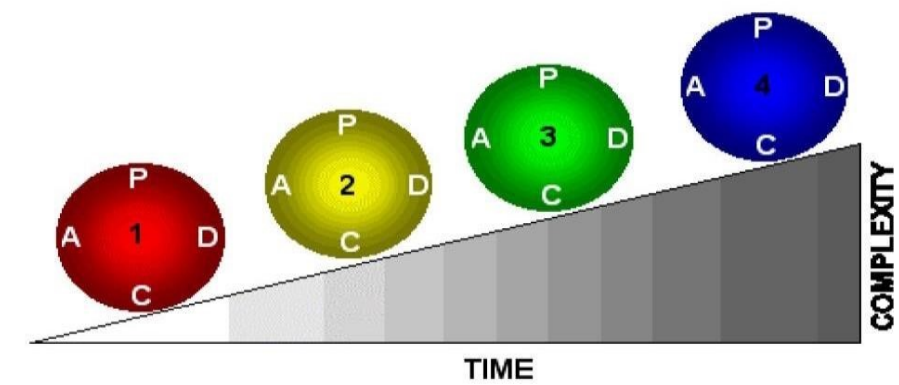

Fonte: Andrade (2005)

\section{MÓDULO PLAN}

PLAN (planejar) é a primeira etapa do método PDCA. É considerado o módulo mais importante por dar início ao ciclo. Em suma, a eficácia futura do ciclo estará baseada num bom planejamento, o qual proverá dados e informações às etapas restantes do método (BADIRU, 1993). Na fase PLAN do ciclo PDCA, todos os envolvidos com o ciclo devem buscar meios a fim de melhorar seus negócios, desenvolvendo assim metas para o funcionamento sistemático da melhoria contínua nesta fase (CLARK, 2001). 
Campos (1996) e Melo (2001) como foi visto uma subdivisão do módulo PLAN em cinco etapas para que ele possa atender a todas as premissas expostas com relação à importância do planejamento dentro do contexto do ciclo.

São as etapas:

1. Localizar o problema;

2. Estabelecer metas;

3. Análise do fenômeno;

4. Análise do processo.

\section{MÓDULO DO}

Na etapa $D O$ (do inglês, executar) deverão ser postos em prática, de acordo com a filosofia de trabalho de cada organização, todos os objetivos e metas traçadas na etapa anterior que já devem estar devidamente formalizadas em um plano de ação. De acordo com Badiru (1993) a eficiência desta etapa está diretamente conectada à existência de plano de ação bem estruturado. A etapa DO permite que o plano de ação seja praticado, organizado em uma escala gradual, de forma a permitir maior eficácia das medidas a serem tomadas.

Uma vez amplamente divulgado e ciente da compreensão de todos os participantes, o plano de ação poderá ser colocado em prática. Durante esta etapa devem-se efetuar verificações periódicas no setor em que as ações estão sendo efetuadas, visando manter o controle e dirimir possíveis dúvidas que possam ocorrer ao longo da execução. Todas as ações tomadas e os resultados obtidos, sejam eles bons ou ruins, devem ser registrados e datados para que alimentem a próxima etapa do ciclo PDCA (etapa CHECK) (ANDRADE, 2003).

As ações pertinentes aos treinamentos de equipe devem ser executadas em primeiro plano, capacitando os funcionários de forma a prepará-los para execução do plano proposto. O funcionário não treinado dificilmente obterá eficácia na realização de alguma ação contida no plano.

\section{MÓDULO CHECK}

O módulo CHECK (do inglês, verificar) do ciclo PDCA é definido por aquele que objetiva a fase de verificação das ações executadas na etapa anterior (DO). Para que a verificação dos resultados na fase em questão seja realizada da maneira mais eficaz possível, os resultados obtidos das ações procedentes à fase de planejamento devem ser devidamente monitorados e formalizados.

Estudos realizados por Clark (2001) com empresas norte americanas que utilizaram métodos PDCA em seus sistemas de gestão indicam que a fase supracitada é a mais importante do ciclo devendo esta ser ressaltada pela organização ou empresa a fim de que seja obtido um resultado satisfatório e eficaz ao final de cada ciclo. "Em um ciclo PDCA forte a etapa CHECK/STUDY é enfatizada" (CLARK, 2001).

A empresa deve atentar aos indicadores propostos na etapa PLAN e monitorados na etapa $D O$ estudando os mesmos minuciosamente, discriminado quais ações obtiveram melhores resultados e quais não alcançaram a eficácia desejada, medidos pelos indicadores em questão. 


\section{MÓDULO ACT}

O módulo $A C T$ é caracterizado pela padronização das ações executadas em $D O$, cujas eficácias já foram verificadas em $C H E C K$. As ações deverão ser baseadas em resultados verificados positivos em CHECK, a fim de padronizar tais ações para que possam ser utilizadas em ocasiões semelhantes (BADIRU, 1993).

O processo, segundo Souza (1997) e Melo (2001), consiste em elaborar um novo padrão ou alterar um já existente. A organização deverá esclarecer no padrão os itens fundamentais de sua estrutura, isto é, "o que" fazer, "quem" deverá executar a ação, "quando" a mesma deverá ser executada, "onde", "como", e principalmente "porque" tal ação será tomada.

Após elaborar os padrões, estes devem ser divulgados por meio de comunicados, reuniões, circulares ou e-mail. Melo (2001) ainda enfatiza que devem ser estabelecidas e amplamente divulgadas a data de início da nova sistemática e quais áreas serão afetadas, para que o novo padrão ocorra ao mesmo tempo e por todos os envolvidos e os locais necessários.

Finalmente, esses padrões devem ser acompanhados regularmente, de modo que a organização possa certificar-se de que eles estão sendo cumpridos. A empresa deve evitar que um problema reapareça por degeneração no cumprimento dos padrões (MELO, 2001).

\section{ESTUDO DE CASO DO PDCA NA PRODUÇÃO DE DOBRADIÇAS}

Na execução do ciclo PDCA, a primeira etapa que será abordada é o Planejamento junto ao $\mathrm{CQ}$, nesta etapa se identifica a problemática e a realização a análise do processo no próprio planejamento conforme a necessidade de melhoria que a empresa necessita. Enquanto em relação à Executar do PDCA, por meio da Folha de Verificação, se buscam informações para o desenvolvimento e execução do plano de ação.

Na Tabela 1 é exemplificadas as informações do P e D.

Tabela 1 - Etapas Planejamento e Execução do PDCA

\begin{tabular}{|c|c|c|c|}
\hline PDCA & FLUXO & ETAPA & OBJETIVO \\
\hline \multirow{4}{*}{$P$} & 1 & Identificação do problema & Peças não conforme e refugos. \\
\hline & 2 & Observação & $\begin{array}{l}\text { Falta de inspeção no processo de } \\
\text { produção dos componentes. }\end{array}$ \\
\hline & 3 & Análise & $\begin{array}{l}\text { O não acompanhamento direto da gestão } \\
\text { de qualidade. }\end{array}$ \\
\hline & 4 & Plano de ação & $\begin{array}{c}\text { Início, meio e fim de cada lote de } \\
\text { produção, rigorosamente pelo inspetor } \\
\text { de qualidade. }\end{array}$ \\
\hline D & 5 & Execução & $\begin{array}{c}\text { Verificação junto ao processo produtivo, } \\
\text { desde a entrada de componentes até sua } \\
\text { montagem final. }\end{array}$ \\
\hline
\end{tabular}

Fonte: Autoria própria (2019)

A primeira avaliação do nosso problema abrange desde origem do método em questão, seus principais envolvidos, até a descrição completa do processo, detalhando cada método de aplicação, envolvendo cada etapa e seus possíveis 
resultados alcançados ao final do ciclo. "O esforço das organizações produtivas em eliminar defeitos no produto evoluiu com a adoção de estratégias gerenciais para otimizar o desempenho dos elementos, equipamentos, pessoas, informações, métodos e materiais que compõe o processo" (PALADINI, 2010).

$\mathrm{Na}$ descrição do processo de montagem é apresentado, conforme o Diagrama 1, o fluxograma no qual estão descritas as etapas de um processo de montagem de dobradiças de pressão, onde as máquinas automatizadas necessitam de apenas uma regulagem para atingir a meta que é de 1.040.000 peças/mensais.

Por sua vez, pela falta de monitoria de um método de qualidade, os componentes da dobradiça produzidos têm uma grande variação de medidas em todos os aspectos prejudicando diretamente a linha de montagem em até $22 \%$.

Pode ser verificado na figura 4 como e o fluxo de trabalho desta operação.

Diagrama 1 - Fluxograma do processo de montagem de dobradiças
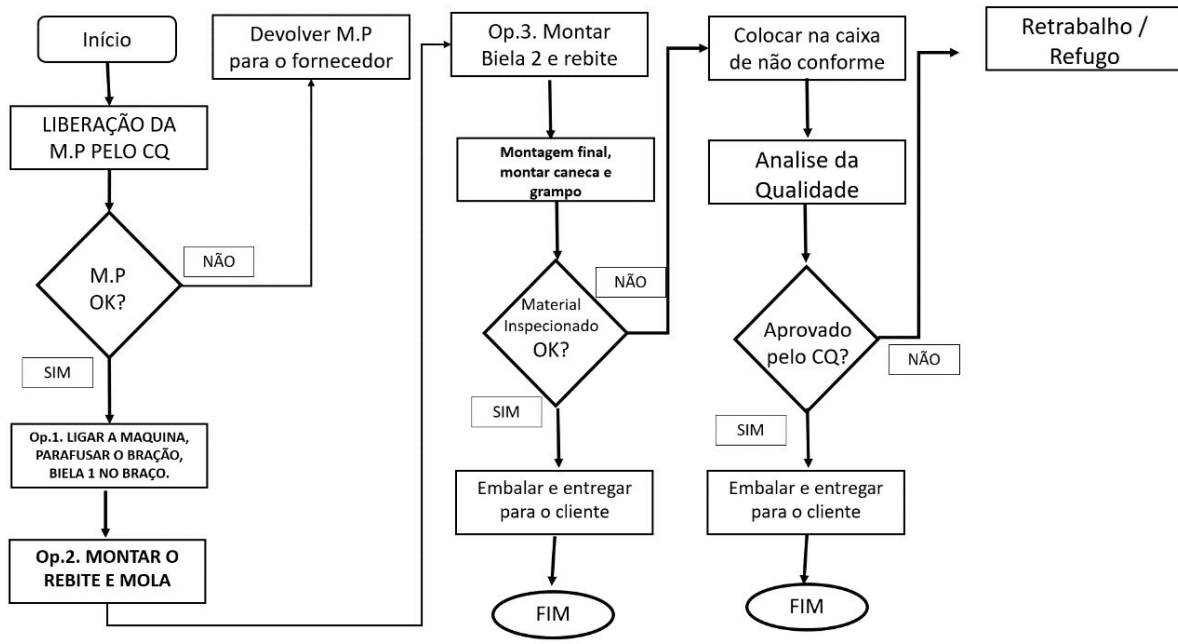

Fonte: Autoria própria (2019)

Também podem ser vistos os dados da Folha de Verificação, por porcentagem de impactos na produção, conforme apresentados no Quadro 1.

Quadro 1 - Dados da Folha de Verificação

\begin{tabular}{|c|c|c|c|c|}
\hline MÊS & $\begin{array}{c}\text { QTD. } \\
\text { FABRICADA }\end{array}$ & $\begin{array}{c}\text { NÃO } \\
\text { CONFORMIDADE }\end{array}$ & $\begin{array}{c}\text { MÉDIA DE NÃO } \\
\text { CONFORMIDADE } \\
\text { (\%) }\end{array}$ & $\begin{array}{c}\text { META } \\
\text { MENSAL }\end{array}$ \\
\hline Abril & 800.800 & 239.200 & 23 & 1.040 .000 \\
\hline Maio & 884.000 & 156.000 & 15 & 1.040 .000 \\
\hline Junho & 936.000 & 104.000 & 10 & 1.040 .000 \\
\hline Julho & 852.800 & 187.200 & 18 & 1.040 .000 \\
\hline Agosto & 811.200 & 228.800 & 22 & 1.040 .000 \\
\hline Setembro & 925.600 & 114.400 & 11 & 1.040 .000 \\
\hline Outubro & 904.800 & 135.200 & 13 & 1.040 .000 \\
\hline Total Geral & 6.115 .200 & 1.164 .800 & 16 & - \\
\hline
\end{tabular}

Fonte: Autoria própria (2019) 
Na terceira etapa de implantação do ciclo PDCA é apresentada a ferramenta Check-list, a qual mostra uma descrição indireta do processo de fabricação, verificando se as ações planejadas foram realizadas de maneira adequada e apontando resultados significativos como a redução de refugos e não conformidades.

O Quadro 2 expõe um exemplo de Check-list de montagem e inspeção para o processo de fabricação.

Quadro 2 - Check list de montagem e inspeção

\begin{tabular}{|c|c|c|}
\hline \multicolumn{2}{|c|}{ SETOR DE DOBRADIÇAS } \\
\hline Etapa & Itens de Verificação & Situação \\
\hline 1 & Mola de pressão & V \\
\hline 2 & Biela interna & V \\
\hline 3 & Biela externa & V \\
\hline 4 & Grampo “U” & V \\
\hline 5 & Rebite & V \\
\hline 6 & Braço (Curvo ou Reto) & V \\
\hline 7 & Caneca & \\
\hline
\end{tabular}

Fonte: Autoria própria (2019)

Após a verificação com o Check-list, ocorreu uma padronização na operação de produção com o ciclo PDCA em que obteve um índice de $80 \%$ concluído, extraindo todos os problemas que prejudicam a produção diretamente.

Na Tabela 2 são evidenciadas as últimas etapas do ciclo PDCA, que seria $C$ (checar) e $A$ (agir), as quais mostram a verificação do $C Q$ e a conclusão do ciclo expondo a eficiência do Método PDCA.

Tabela 2 - Etapas Verificação e Ação do PDCA

\begin{tabular}{ccl} 
PDCA & FLUXO & AÇÃO \\
C & 6 & $\begin{array}{l}\text { Verificar se a dobradiça está montada corretamente, se } \\
\text { atentando aos mínimos detalhes. }\end{array}$ \\
& 7 & $\begin{array}{l}\text { Padronizar o método de montagem e fabricação de todos } \\
\text { os componentes da dobradiça. }\end{array}$ \\
A & 8 & $\begin{array}{l}\text { Aguardar a aprovação do Controle de Qualidade (CQ) para } \\
\text { seguir para o setor de embalagem. }\end{array}$ \\
\hline
\end{tabular}

Fonte: Autoria própria (2019) 


\section{RESULTADOS}

Com base no estudo de caso apresentado neste trabalho, o Gráfico 1 exemplifica a produção em \% sem o acompanhamento do ciclo PDCA, o qual mostra a variação de peças em percentual de não conformidade dos componentes de dobradiças, mostrando assim a necessidade de melhoria para alcançar a meta de produção mensal determinada pela empresa

Gráfico 1 - Demonstrativo de não conformidades

Produçāo de componetes para dobradiças semestral

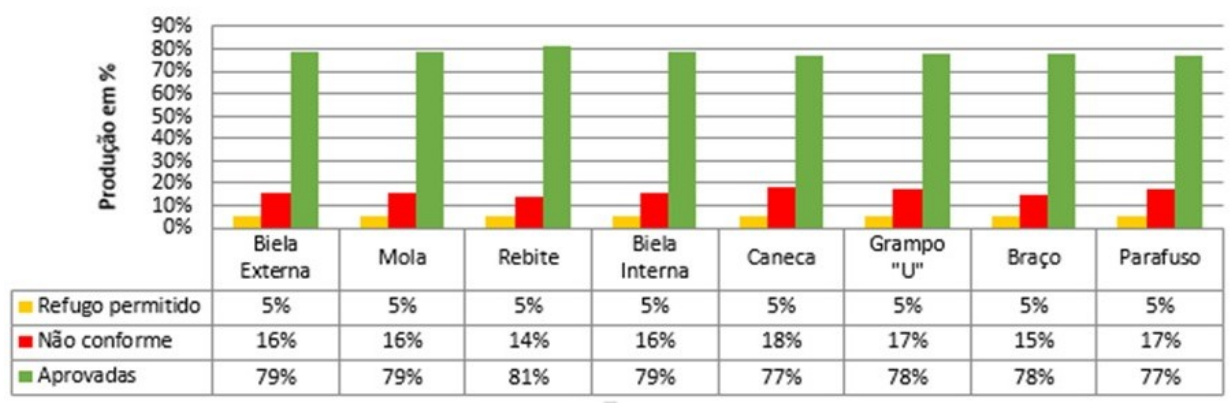

Fonte: Autoria própria (2019)

O Gráfico 2 apresenta os demonstrativos de dobradiças montadas em que apresenta o índice de não conformidades, que afetará diretamente a produção mensal de peças montadas, por sua vez o índice de peças não conforme não indica refugo e sim retrabalho, pois os componentes produzidos com até $20 \%$ fora do especificado ainda irá para a linha de montagem, mas em segundo plano pelo fato de estar fora do especificado a montadora diminuirá seu ritmo, com tudo, se não obtiver resultados significativos desta vez gerará refugos.

Gráfico 2 - Demonstrativos de dobradiças montadas semestralmente

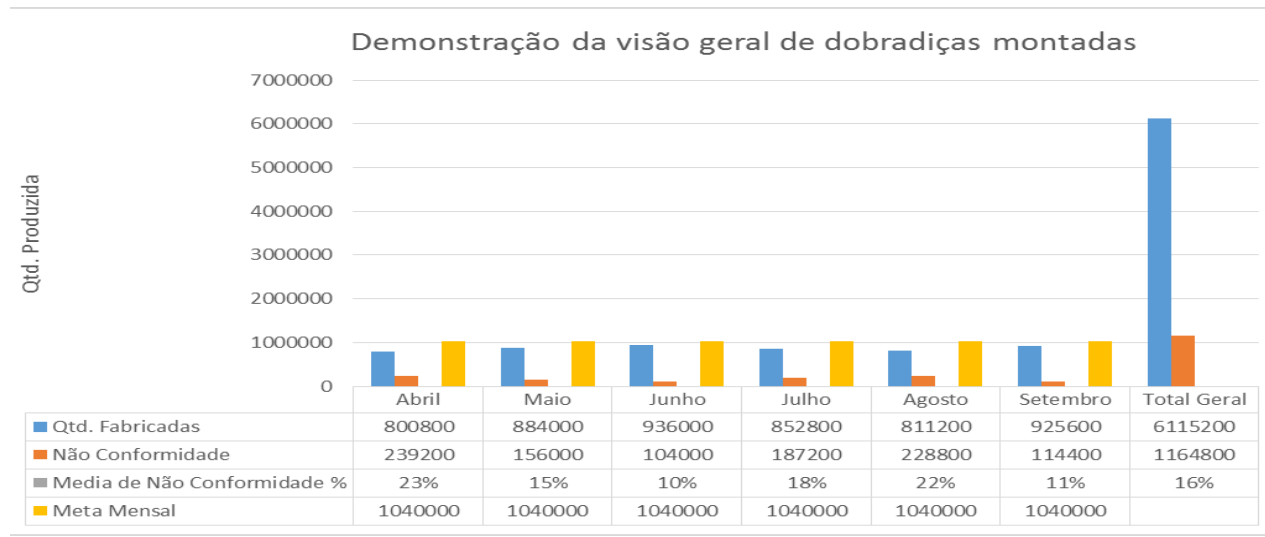

Fonte: Autoria própria (2019)

Com a implantação do método PDCA, se nota um aumento significativo de peças montadas e a diminuição de índice de não conformidade mantendo assim uma média de $5 \%$ de refugos e não conformidade, desta forma se obtém uma porcentagem estável na produção de componentes para dobradiças. 
Assim sendo, a implantação do ciclo PDCA afeta diretamente a linha de montagem do produto, o ciclo proporciona ótima fabricação dos componentes e por consequência uma excelente produção de dobradiças atingindo o principal objetivo que é a meta mensal.

O Rendimento da produção aumentou em peças produzidas com qualidade e consequentemente a não conformidade zerou, considerando o índice de 5\% para refugos e não conformidades, conforme exposto pelo Gráfico 3.

Gráfico 3 - Demonstrativos de Resultados

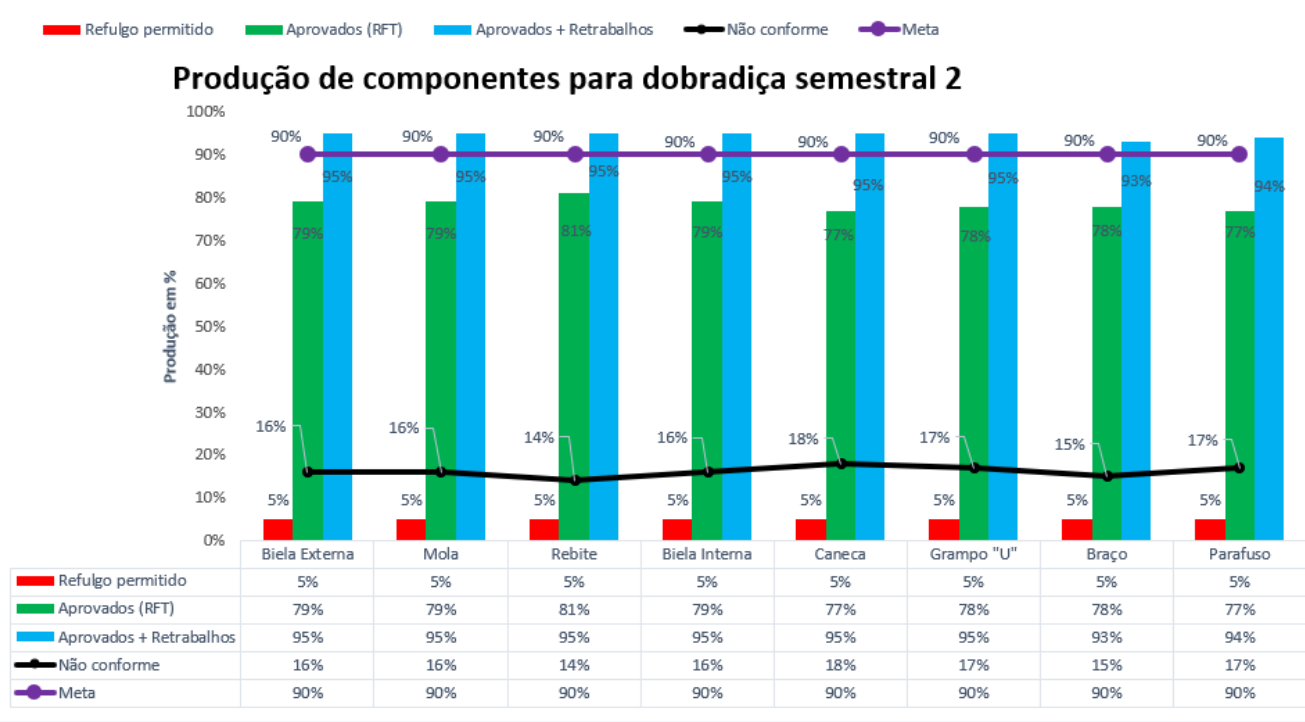

Fonte: Autoria própria (2019)

\section{CONCLUSÃO}

De acordo com os dados apresentados neste artigo, obteve-se à conclusão de que a implementação do ciclo PDCA para melhoria na linha de produção de uma indústria fabricante de dobradiças que atende o setor moveleiro, será extremamente positiva. Pois, a produção da empresa passará a atingir sua meta e reduzirá significativamente o índice de refugos e retrabalhos, gerando assim economia no seu processo produtivo.

Neste estudo de caso foram realizadas análises dos dados coletados através de ferramentas da qualidade como a folha de verificação e o check list, que mostraram a necessidade da utilização do ciclo PDCA para a empresa estudada, tendo como principal desafio será demonstrar à empresa a necessidade de ter o PDCA como ajuda neste segmento, pois trata-se de uma empresa com conceito "fechado", que conserva os hábitos de produção como antigamente.

Sempre há algo para ser melhorado, se acredita que a implementação do ciclo PDCA nesta empresa trará novas oportunidades de melhorias em outros processos, desde a produção até a administração dela, ocasionando assim o crescimento de mercado. 


\title{
PDCA application in the production process of hinges
}

\begin{abstract}
Due to the evolution in production systems, due to technological advances and improvement in quality systems, it is possible to realize that over the years, many companies have invested in high technology machinery. However, sometimes some of these companies forget to use a quality tool to monitor their production processes, which is extremely important to measure their performance and identify possible improvements in their process. Therefore, the company that invested in automation technology focused on hinge assembly was chosen as the basis for this case study, which as well as other furniture industries also joined the automation in the production line. Aiming at the assembly process, it was identified that even with technological investments, it is necessary to insert a quality tool to follow the processes. Thus, through demonstrations of viability in relation to increased production will be exposed to the efficiency of a quality tool. In a general study, the objectives to be achieved with QA automation and monitoring could fall into two major problems, such as scrap and rework.
\end{abstract}

KEYWORDS: PDCA. Hinge Production. PDCA Check List. PDCA Processes. PDCA Check Sheet. 


\section{REFERÊNCIAS}

AGUIAR, Silvio. Integração das ferramentas da qualidade ao PDCA e ao programa seis sigma. Belo Horizonte: Editora de Desenvolvimento Gerencial, 2002.

ANDRADE, Fábio Felippe de. 0 método de melhorias PDCA. 2003. Tese de Doutorado. Universidade de São Paulo.

ANDRADE, Maria Margarida; DE ANDRADE MARTINS, João Alcino. Introdução à metodologia do trabalho científico: elaboração de trabalhos na graduação. Atlas, 2005.

BADIRU, Adedeji Bodunde et al. Practitioner's guide to quality and process improvement. Chapman \& Hall, 1993.

CAMPOS, Vicente Falconi. Gerenciamento da rotina do trabalho do dia-a-dia. INDG Tecnologia e Serviços, 2004.

CLARK, A. B. How managers can use the Shewhart PDCA Cycle to get better results. Houston: Jesse H. Jones Scholl of Business-Texas Southern University, 2001.

GORINI, Ana Paula Fontenelle. Panorama do setor moveleiro no Brasil, com ênfase na competitividade externa a partir do desenvolvimento da cadeia industrial de produtos sólidos de madeira. Rio de Janeiro: BNDES, v. 199, 1998. Disponível em: http://www.bndes.gov.br/SiteBNDES/bndes/bndes_pt/Galerias/Convivencia/Pub licacoes/Consulta_Expressa/Setor/Produtos_Florestais/199809_1.html. Acesso em 5 de outubro de 2019.

MELO, Celso Pinto; CARAMORI, E. J. PDCA Método de melhorias para empresas de manufatura-versão 2.0. Belo Horizonte: Fundação de Desenvolvimento Gerencial, 2001.

NASCIMENTO, Adriano Fagner Gonçalves. A utilização da metodologia do ciclo PDCA no gerenciamento da melhoria contínua. Minas Gerais: ICAP, 2011.

NOGUEIROL, R. L. Gestão da Qualidade. 1. São Paulo: Érica, 2010.

PALADINI, Edson Pacheco. Gestão da qualidade: teoria e prática. In: Gestão da qualidade: teoria e prática. 2010. 
SLACK, Nigel et al. Administração da produção. São Paulo: Atlas, 2009.

SOUZA, Roberto de; ABIKO, Alex. Metodologia para desenvolvimento e implantação de sistemas de gestão da qualidade em empresas construtoras de pequeno e médio porte. São Paulo, v. 335, 1997.

VEYRAT, Pierre. Ciclo PDCA: conceito determinante na melhoria de processos. Disponível em: https://www.venki.com.br/blog/ciclo-pdca-conceito/. Acesso em: 5 de outubro de 2019

CRUZ FILHO, A.P. et al. Aplicação de PDCA no processo produtivo de dobradiças. R. Gest. Industr., Ponta Grossa, v. 16, n. 3, p. 64-75, Jul./Set. 2020. Disponível em: https://periodicos.utfpr.edu.br/revistagi Correspondência:

Almir Pereira da Cruz Filho

Centro Universitário ENIAC (ENIAC), Guarulhos, São Paulo, Brasil.

Direito autoral: Este artigo está licenciado sob os termos da Licença Creative Commons-Atribuição 4.0 Internacional.

\section{(c) (1)}

